\title{
THE FREE OSCILLATIONS OF A BUCKLED PANEL*
}

\author{
BY \\ W. D. HAYES \\ Princeton University \\ AND \\ J. W. MILES \\ University of California**
}

Summary. The non-linear equations of motion of a buckled, two-dimensional panel are formulated in dimensionless form. A Fourier expansion in the space variable is introduced and an approximate solution is obtained for the period of free oscillation on the assumption of only two degrees of freedom, one of which is eliminated by the buckling constraint. The periods of the simplest asymmetric and symmetric modes are plotted as a function of the energy level. It is found that for very small energy levels the period of the buckled panel lies between the periods of the (two) unbuckled degrees of freedom. As the energy is increased the period approaches infinity at some critical energy level and thereafter decreases monotonically.

1. Introduction. The subject of this study is the oscillation of a two-dimensional, buckled plate. The problem is of considerable practical interest and serves as a simple model of the phenomenon often described as "oil canning." Moreover, it is of some theoretical interest that the original partial differential equation of motion is linear and that the deflection is required to be small but that the problem is nevertheless non-linear in consequence of the buckling constraint.

The rather more difficult problem of the aerodynamic excitation of a buckled plate has been studied previously ${ }^{1,2}$, but the simplicity of the present problem allows a more complete solution. In particular, only the free oscillations will be treated, whereas self-excited oscillations are possible under aerodynamic excitation.

2. Equations of motion. Consider a two-dimensional plate of flexural stiffness $D$ and mass $m$ per unit area that is loaded axially by two equal and opposite compressive forces $F(t)$ per unit width, applied at the pin supports $x=0$ and $x=l$. The coordinate $x$ is measured along the plate from the left support, while $y$, the displacement, is measured positive up, as shown in Fig. $1 ; t$ is the time. The equation of motion of the plate then reads

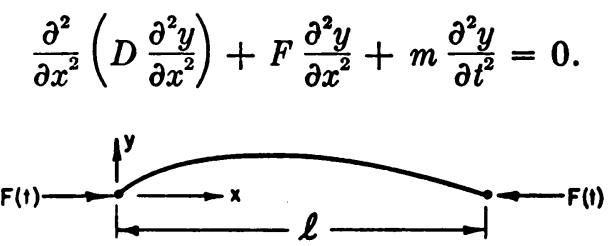

Fig. 1. Two-dimensional buckled plate.

*Received January 31, 1955.

**The present work was carried out while the latter author was a Consultant to the Douglas Aircraft Co., Santa Monica, Calif., and appeared as Douglas Report SM-18439.

${ }^{1}$ W. D. Hayes, $A$ buckled plate in a supersonic stream, No. Amer. Avia. Rep. AL-1029, Downey, Calif., 1950.

2J. W. Miles, Dynamic chordwise stability, No. Amer. Avia. Rep. AL-1140, Downey, Calif., 1950. 
In general, $D$ and $m$ may be functions of $x$, but in the following they will be assumed to be constant.

It is required to obtain a solution to (2.1) subject to the boundary conditions of zero displacement and zero bending moment at the ends, viz.

$$
\begin{gathered}
y(0)=y(l)=0, \\
y_{x z}(0)=y_{x x}(l)=0,
\end{gathered}
$$

and, in addition, to the constraint that the plate be buckled such that its length has the constant value $l+\delta l$. It will be assumed that $\delta$, the fraction of the length by which the plate is buckled, is small, whence the deflections also must be small; then, by definition,

$$
\delta=\frac{1}{l}\left[\int_{0}^{l}\left[1+\left(\frac{\partial y}{\partial x}\right)^{2}\right]^{1 / 2} d x-l\right] \bumpeq \frac{1}{2 l} \int_{0}^{l}\left(\frac{\partial y}{\partial x}\right)^{2} d x
$$

In order to render the formulation dimensionless, the following dimensionless quantities and characteristic measures are introduced:

$$
\begin{aligned}
\xi & =x / l_{0}, \\
\eta & =y / l_{0}=\eta(\xi, \tau), \\
\tau & =t / t_{0}, \\
f(t) & =F(t) / F_{0}, \\
l_{0} & =l / \pi, \\
t_{0} & =(l / \pi)^{2}(m / D)^{1 / 2}, \\
F_{0} & =(\pi / l)^{2} D .
\end{aligned}
$$

The reference length, $l_{0}$, is chosen in anticipation of the Fourier expansion in $\xi$ introduced below (Sec. 3). The reference time, $t_{0}$, is the reciprocal of the fundamental (circular) frequency of simple harmonic motion for the plate in the absence of the buckling loads. $F_{0}$ is the Euler load, i.e., the smallest axial load required to produce static buckling in the absence of normal loading.

Introducing the dimensionless variables $\xi, \eta, \tau$, and $f$ in the original formulation of (1)-(3) leads to

$$
\begin{gathered}
\frac{\partial^{4} \eta}{\partial \xi^{4}}+f(\tau) \frac{\partial^{2} \eta}{\partial \xi^{2}}+\frac{\partial^{2} \eta}{\partial \tau^{2}}=0, \quad \eta=\eta(\xi, \tau), \\
\eta(0, \tau)=\eta(\pi, \tau)=0, \\
\eta_{\xi \xi}(0, \tau)=\eta_{\xi \xi}(\pi, \tau)=0, \\
\frac{1}{2 \pi} \int_{0}^{\pi}\left(\frac{\partial \eta}{\partial \xi}\right)^{2} d \xi=\delta .
\end{gathered}
$$

3. Fourier series reduction. In order to eliminate the space variable from (2.11) and (2.13) it is convenient to expand $\eta(\xi)$ in a set of functions that individually satisfy the boundary conditions (2.12). (Such an expansion is permissible in virtue of the linearity of (2.11) in $\eta$ as a function of $\xi$.) It is evident that the required set is furnished by

$$
\eta_{n}(\xi)=\sin (n \xi), \quad n=1,2, \cdots .
$$


Accordingly, the Fourier expansion

$$
\eta(\xi, \tau)=\sum_{n=1}^{N} a_{n}(\tau) \sin (n \xi)
$$

is posed.

Introducing (2) in (2.11) and (2.13) and denoting differentiation with respect to $\tau$ by dots yields

$$
\begin{aligned}
& a_{n}^{\ddot{*}(\tau)}+\left[n^{4}-n^{2} f(\tau)\right] a_{n}(\tau)=0, \quad n=1,2, \cdots, N \\
& \frac{1}{4} \sum_{1}^{N} n^{2} a_{n}^{2}=\delta
\end{aligned}
$$

which constitute a set of $N+1$ equations for the determination of the $N+1$ unknowns $a_{1}, a_{2}, \cdots a_{N}, f$.

The initial values of $\eta$ and $\eta_{r}$ are

$$
\begin{aligned}
& \eta(\xi, 0)=\sum_{n=1}^{N} a_{n}(0) \sin (n \xi), \\
& \eta_{\tau}(\xi, 0)=\sum_{n=1}^{N} a_{n}(0) \sin (n \xi) .
\end{aligned}
$$

In principle, $N$ must be infinite for arbitrarily prescribed initial values of $\eta$ and $\eta_{r}$, but, whatever the finite value of $N$ that will permit a satisfactory approximation to these initial values, they must satisfy (3.4) and its time derivative, viz.

$$
\begin{gathered}
\sum_{1}^{N} n^{2} a_{n}^{2}(0)=4 \delta, \\
\sum_{1}^{N} n^{2} a_{n}(0) a_{n}^{\cdot}(0)=0 .
\end{gathered}
$$

It is clear that the minimum number of modes required to satisfy (6a) and (6b) in a non-trivial way is 2 , a single mode leading only to a purely static solution.

It does not appear possible to obtain an explicit, general solution to the problem formulated in (3)-(5). Accordingly, the analysis will be restricted to the simplest possible situation, where only two (Fourier) modes are required, say $n=1$ and $n=N(N \geq 2)$. For definiteness it will be assumed that $a_{N}(0)=0$ (although this limitation can be removed simply by changing the origin of time) and the initial conditions

$$
\begin{gathered}
a_{1}(0)=2 \delta^{1 / 2}, \quad a_{N}(0)=0, \\
a_{1}(0)=0, \quad a_{N}(0)=2(\delta w)^{1 / 2},
\end{gathered}
$$

posed, where, as will be brought out in more detail below, $w$ defines the energy level of the oscillation. It should be emphasized that this reduced two degree of freedom problem does not afford a general solution inasmuch as it is not possible to superpose solutions for different $N$.

4. Energies. Before attempting an approximate solution to the problem formulated in the preceding section, it is instructive to consider the various energies involved. Inasmuch as the buckled state of the plate stands as a central feature of the analysis, 
an appropriate reference quantity or unit of measurement is the work required to buckle the plate in the absence of normal loading, namely

$$
W_{0}=\left(\frac{\pi^{2} D}{l^{2}}\right) \delta l
$$

The potential and kinetic energies per unit width of the plate are given by

$$
\begin{aligned}
& K=\frac{1}{2} \int_{0}^{l} m\left(\frac{\partial y}{\partial t}\right)^{2} d x \\
& V=\frac{1}{2} \int_{0}^{l} D\left(\frac{\partial^{2} y}{\partial x^{2}}\right)^{2} d x
\end{aligned}
$$

Introducing the dimensionless notation of (2.4)-(2.10) and (4.1), these energies can be placed in the dimensionless forms

$$
\begin{aligned}
& k=K / W_{0}=\frac{1}{2 \pi \delta} \int_{0}^{\pi}\left(\frac{\partial \eta}{\partial \tau}\right)^{2} d \xi \\
& v=V / W_{0}=\frac{1}{2 \pi \delta} \int_{0}^{\pi}\left(\frac{\partial^{2} \eta}{\partial \xi^{2}}\right)^{2} d \xi
\end{aligned}
$$

Introducing the Fourier expansion of (3.2) in (4) and (5) yields

$$
\begin{aligned}
& k(\tau)=\frac{1}{4 \delta} \sum_{1}^{N}\left[a_{n}^{\cdot}(\tau)\right]^{2}, \\
& v(\tau)=\frac{1}{4 \delta} \sum_{1}^{N} n^{4} a_{n}^{2}(\tau) .
\end{aligned}
$$

Imposing the requirement of conservation of energy, viz.

$$
k+v=C
$$

then yields

$$
\sum_{1}^{N}\left(a_{n}^{.9}+n^{4} a_{n}^{2}\right)=C .
$$

The constant $C$ is determined by the initial energy in the system. For the two degree of freedom system having the initial conditions (3.7)

$$
\left(a_{1}^{. \prime}+a_{N}^{.3}\right)+\left(a_{1}^{2}+N^{4} a_{N}^{2}\right)=4 \delta(1+w),
$$

where $w$ is defined as the (dimensionless) energy in excess of the work required to produce the static configuration.

Alternatively, (9) can be obtained by multiplying both sides of (3.3) by $a_{n}^{\cdot}$, summing over $n$, imposing the constraint that the derivative of (3.4) with respect to $\tau$ vanish identically, and integrating the remainder with respect to $\tau$.

5. Two mode solution. Returning to the solution of the two mode problem having the initial conditions of (3.7), the resulting equations of motion then can be obtained either from (3.3) and (3.4) or (4.10) and (3.4). The latter automatically eliminates the unknown $f(\tau)$, which, however, can be determined from (3.3) once $a_{1}(\tau)$ and $a_{N}(\tau)$ are known. 
The buckling constraint (3.4) will be satisfied by writing

$$
\begin{aligned}
a_{1}(\tau) & =2 \delta^{1 / 2} \cos [\theta(\tau)], \\
N a_{N}(\tau) & =2 \delta^{1 / 2} \sin [\theta(\tau)] .
\end{aligned}
$$

Substituting these expressions in (4.10) yields

$$
\left[1+\left(N^{2}-1\right) \sin ^{2} \theta\right]\left[1+\left(\theta^{*} / N\right)^{2}\right]-1=w .
$$

It may be remarked that, by definition, $w$ vanishes for the static configuration $(\theta \equiv 0)$.

In order to determine the period of the free oscillation of the plate as a function of its energy level, (2) is first solved for $\theta^{\circ}$, viz.

$$
\theta^{\cdot}= \pm N\left[\frac{w-\left(N^{2}-1\right) \sin ^{2} \theta}{1+\left(N^{2}-1\right) \sin ^{2} \theta}\right]^{1 / 2} .
$$

It is evident that the phase plane $\left(\theta^{\circ}, \theta\right)$ trajectories are closed, quasielliptic, curves for $w<\left(N^{2}-1\right)$ and single valued, periodic curves for $w>\left(N^{2}-1\right)$. The critical trajectory $w=\left(N^{\wedge}-1\right)$ is designated as the "separatrix." (The terminology is that of Minorsky ${ }^{3}$.) These topological distinctions are intimately connected with the singularities of the differential equation satisfied by the phase plane trajectories, namely [from logarithmic differentiation of (5.2)]

$$
\frac{\theta^{\circ} d \theta^{\circ}}{\left(N^{2}-1\right)\left(N^{2}+\theta^{\cdot 2}\right)}=\frac{-\sin \theta \cos \theta d \theta}{\left[1+\left(N^{2}-1\right) \sin ^{2} \theta\right]} \text {. }
$$

It is seen that the singular points (at which $d \theta^{\circ} / d \theta$ is indeterminate) arise at $\theta=s \pi / 2$ where $s$ is any integer. Even values of $s$ correspond to saddle points (points of stable, static equilibrium), in the neighborhood of which the trajectories defined by (3) are elliptic and $w \ll 1$. Odd values of $s$ correspond to vortex points (points of unstable static equilibrium), in the neighborhood of which the trajectories are hyperbolic and $\left|w-\left(N^{2}-1\right)\right| \ll 1$. The separatrix passes through these vortex points and in this neighborhood coincides with the asymptotes of the hyperbolic trajectories.

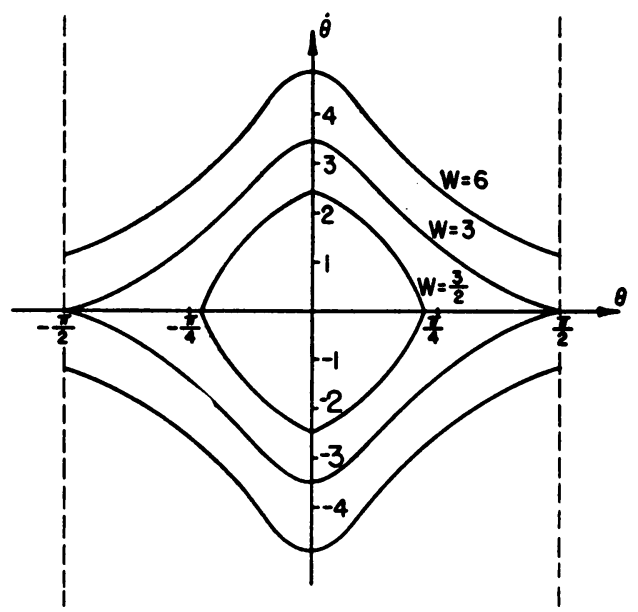

Fig. 2. Phase plane trajectories of free oscillations $(N=2)$.

${ }^{3}$ N. Minorsky, Non-linear mechanics, Edwards Bros., Ann Arbor, 1947. 
Three typical trajectories obtained by assigning the values $w=3 / 2,3$ and 6 and $N=2$ are plotted in Fig. 2. The pattern is, of course, repeated in each $\pi$ interval of $\theta$.

The three possible types of oscillations under consideration are somewhat analogous to the "wig-wag" oscillations of a pendulum (total energy $<2 m g l$ ), the "loop the loop" oscillations (total energy $>2 \mathrm{mgl}$ ) and the critical case where the total energy $(=2 \mathrm{mgl}$ ) is just sufficient to invert the pendulum. By analogy with this classical problem, we may expect the period of oscillation of the buckled plate to increase from its initial value at $w=0$ to infinity at $w=N^{2}-1$ and then to decrease monotonically as $w$ is increased beyond the critical value. As in the case of the pendulum, the maximum possible potential energy of the system $\left(v=N^{2}\right.$, of which 1 is already present in consequence of the buckling) is attained for $w=N^{2}-1$, and additional energy can appear only in kinetic form, whence the above inference of a monotonic decrease in the period.

It remains to integrate (3). If $w<N^{2}-1$ the period is defined as the time required to cover the complete orbit in the phase plane, which, in virtue of symmetry, is given by

$$
T_{a}=4 \int_{0}^{\sin ^{-1}\left\{w /\left(N^{2}-1\right)\right\}^{1 / 0}} \frac{d \theta}{\theta^{*}(\theta)}, \quad w<N^{2}-1 .
$$

If $w>N^{2}-1$, the time required for a given configuration to be reestablished is evidently

$$
T_{b}=4 \int_{0}^{\pi / 2} \frac{d \theta}{\theta^{*}(\theta)}, \quad w>N^{2}-1 .
$$

In the first case it is convenient to introduce the change of variable

$$
\sin \theta=\left(\frac{w}{N^{2}-1}\right)^{1 / 2} \sin \alpha
$$

which, after substituting $\theta^{\circ}$ from (3), reduces (5a) to

$$
T_{a}=\frac{4}{N} \int_{0}^{\pi / 2}\left[\frac{1+w \sin ^{2} \alpha}{\left(N^{2}-1\right)-w \sin ^{2} \alpha}\right]^{1 / 2} d \alpha .
$$

The direct substitution of $\theta^{\circ}$ in $(5 \mathrm{~b})$ yields

$$
T_{b}=\frac{4}{N} \int_{0}^{\pi / 2}\left[\frac{1+\left(N^{2}-1\right) \sin ^{2} \theta}{w-\left(N^{2}-1\right) \sin ^{2} \theta}\right]^{1 / 2} d \theta .
$$

Following standard procedures, each of the integrals in (7) reduces to an elliptic integral of the third kind, the end results being

$$
\begin{gathered}
T_{a}=\frac{4}{N}\left(\frac{1+w}{N^{2}-1}\right)^{1 / 2} I\left[\frac{N}{\left(N^{2}-1\right)^{1 / 2}}\left(\frac{w}{1+w}\right)^{1 / 2},\left(\frac{w}{N^{2}-1}\right)^{1 / 2}\right], \\
T_{b}=4 w^{-1 / 2} I\left[\frac{\left(N^{2}-1\right)^{1 / 2}}{N}\left(\frac{1+w}{w}\right)^{1 / 2},\left(\frac{N^{2}-1}{w}\right)^{1 / 2}\right], \\
I(k, \beta)=\int_{0}^{x / 2} \frac{\left(1-k^{2} \sin ^{2} \varphi\right)^{1 / 2}}{\left(1-\beta^{2} \sin ^{2} \varphi\right)} d \varphi .
\end{gathered}
$$


(It is of interest to remark that the arguments of $I$ in (8a) and (8b) are the reciprocals of one another.) The integral $I$ is evaluated $a^{4}$

$I(k, \beta)=K(k)+\frac{1}{\beta}\left(\frac{k^{2}-\beta^{2}}{1-\beta^{2}}\right)^{1 / 2}\left\{E(k) F\left[k, \sin ^{-1}(\beta / k)\right]-K(k) E\left[k, \sin ^{-1}(\beta / k)\right]\right\}$.

If $w$ approximates the critical value $\left(N^{2}-1\right)(5.10)$ is indeterminate, but a direct reduction of either of (5.8) yields

$$
T=\frac{2}{\left(N^{2}-1\right)^{1 / 2}} \ln \left|\frac{4\left(N^{2}-1\right)}{w-\left(N^{2}-1\right)}\right|+O\left[w-\left(N^{2}-1\right)\right] .
$$

In the limiting cases of very small or very large values of $w$ Eqs. (7) reduce to

$$
\begin{aligned}
& T_{a}=\frac{2 \pi}{N\left(N^{2}-1\right)^{1 / 2}}\left[1+\frac{1}{4}\left(\frac{N^{2}}{N^{2}-1}\right) w\right]+O\left(w^{2}\right), \\
& T_{b}=4 E\left[\frac{\left(N^{2}-1\right)^{1 / 2}}{N}\right] w^{-1 / 2}+O\left(w^{-3 / 2}\right) .
\end{aligned}
$$

Now, in the absence of buckling, the periods of the first and $N$ th modes of natural oscillation would be [set $\delta=f=b_{n}=0$ in (3.3) and (3.4)] $2 \pi$ and $2 \pi / N^{2}$, and $T_{a}$ lies between these values in the limit $w=0$, although closer to the second than the first. This rather curious state of affairs, which, however, is typical of non-conservative systems, is to be

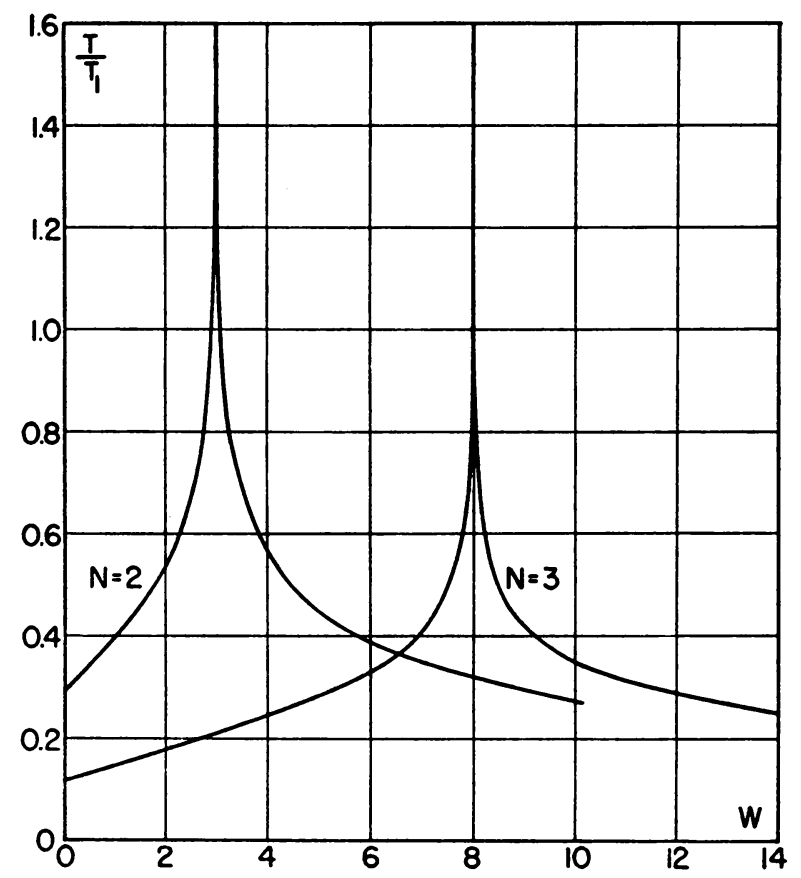

Fig. 3. The period $(T)$ of free oscillation of a buckled plate, referred to the fundamental period $\left(T_{1}\right)$ of the unbuckled plate.

${ }^{4}$ D. Bierens de Haan, Nouvelles tables d'integrales definies, G. E. Stechert \& Co., New York, 1939, Sec. 57(1) and Sec. 61(8). 
contrasted with the coupled oscillations of a linear two degree of freedom system, where the resulting period cannot be between the periods of the uncoupled oscillations.

The period obtained by dividing (5.8)-(5.12) by $2 \pi$ (so that unity corresponds to the fundamental period of the unbuckled plate) is plotted $v s . w$ in Fig. 3 for the two most important possibilities $N=2$ (simplest asymmetric) and $N=3$ (simplest symmetric). The reciprocal of this period, being the ratio of the frequency of oscillation to that for the unbuckled plate, is plotted in Fig. 4.

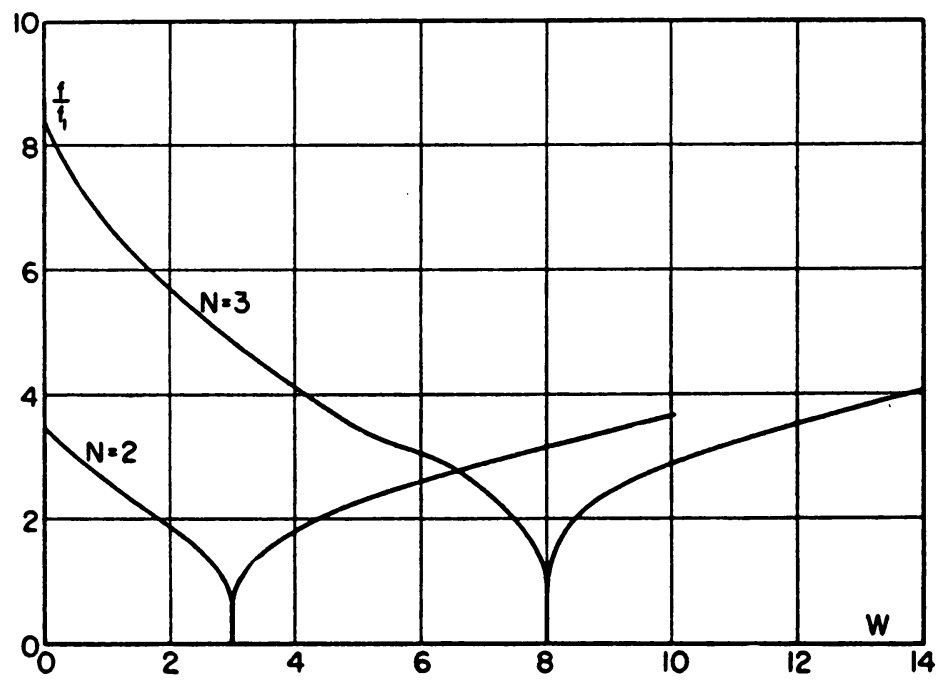

Fra. 4. The frequency $(f)$ of free oscillation of a buckled plate, referred to.the fundamental frequency $\left(f_{1}\right)$ of the unbuckled plate. 